\title{
Prognostic value of carbonic anhydrase VII expression in colorectal carcinoma
}

\author{
Guang-Zhen Yang ${ }^{1,2+}$, Liang Hu ${ }^{1 *+}$, Jian Cai ${ }^{3 \dagger}$, Hai-Yang Chen ${ }^{4 \dagger}$, Yu Zhang ${ }^{1}$, Dan Feng ${ }^{5}$, Chen-Ye Qi ${ }^{1}$, Yan-Xia Zhai ${ }^{1}$, \\ Hui Gong ${ }^{1}$, Hao Fu', Qing-Ping $\mathrm{Ca}^{16^{* *}}$ and Chun-Fang Gao ${ }^{1 *}$
}

\begin{abstract}
Background: Carbonic anhydrases (CAs) have been implicated in the pathogenesis of human cancers. Carbonic anhydrase VII (CA7), a member of the CA gene family, was recently demonstrated to be expressed in several human tissues including colon. Nevertheless, the expression and clinical relevance of CA7 in colorectal carcinoma (CRC) has not been investigated.
\end{abstract}

Methods: Real-time PCR, western blot, and immunohistochemistry analyses were used to determine CA7 expression in CRC clinical samples. The correlation of CA7 expression with clinicopathologic features was assessed in 228 patients from Luoyang, China (training cohort) and validated in 151 patients from Shanghai, China (validation cohort). Kaplan-Meier and Cox proportional regression analyses were used to estimate the association between CA7 expression and patients' survival.

Results: CA7 expression was frequently downregulated in CRC tissues at both the mRNA and protein levels. Reduced expression of CA7 was significantly correlated with poor differentiation, positive lymph node metastasis, advanced TNM stage and unfavorable clinical outcome not only in the training cohort but also in the validation set. Survival analysis indicated that patients with lower CA7 expression had a significantly shorter disease-specific survival (DSS) than those with higher CA7 expression. Importantly, further stage-based analyses revealed that decreased CA7 expression significantly predicted poor DSS and was an independent adverse prognostic indicator for patients with early stage tumors in both cohorts.

Conclusions: Our results indicate that decreased expression of CA7 correlates with disease progression and predicts poor prognosis in CRC, especially for patients with early stage tumors.

Keywords: CA7, Colorectal carcinoma, Survival, Prognosis, Early stage

\section{Background}

Colorectal carcinoma (CRC) is one of the leading causes of cancer-related death globally, accounting for more than 1.2 million new cases and 600,000 deaths per year $[1,2]$. Although the survival of patients with CRC has slowly but steadily improved during the past decades in the developed countries, mortality rates have continued increasing in countries including China [3,4]. Due to post-surgical recurrence and fatal distant metastasis, the

\footnotetext{
*Correspondence: Ihu@outlook.com; caiqingpingwcwk@163.com; chunfgao@163.com

${ }^{\dagger}$ Equal contributors

'Anal-Colorectal Surgery Institute, 150th Hospital of PLA, Luoyang, China ${ }^{6}$ Department of Gastrointestine Surgery, Changzheng Hospital, Second Military Medical University, Shanghai, China

Full list of author information is available at the end of the article
}

prognosis for CRC patients has shown only limited improvement despite advances in treatment approaches over the last few years. Therefore, it is urgent needed to search for valuable biomarkers to improve prognosis prediction and clinical outcome of patients with CRC.

Carbonic anhydrases (CAs) are a family of ubiquitously expressed metalloenzymes that catalyze the reversible conversion of carbon dioxide to bicarbonate and proton [5]. Previously studies have revealed that CAs are involved in multiple physiological and pathological processes including gluconeogenesis, lipogenesis, ureagenesis and tumorigenicity [6]. In humans, at least $15 \mathrm{CA}$ isozymes with different catalytic activity, subcellular localization and tissue distribution have been described [5]. Among them, aberrant expression 
of CA I, II, IX, XII and XIII has been reported in CRC [7-12]. Recently, CA7, a cytosolic isoform of CAs with high carbon dioxide hydration activity, was demonstrated to be expressed in several normal tissues including colon [13]. A previous work from a gene expression microarray analysis revealed that CA7 was downregulated in clinically left sided colon tumors [14]. More recently, a bioinformatics-based study indicated CA7 as an important suppressor gene for the classification of normal and CRC tissues [15]. In addition, it has been shown that upregulated expression of CA7 was associated with poor prognosis of patients with astrocytomas [16].

Despite these, to our knowledge, systematic investigation of the expression and clinical implications of CA7 in human CRC has not been reported. In the present study, we examined the expression of CA7 in CRC clinical samples and assessed the correlation of CA7 expression with clinicopathologic features and with patient survival in a training cohort and further validated our findings in an independent external cohort. Our data demonstrated that decreased expression of CA7 could serve as an independent predictor of poor prognosis in CRC, especially for patients who have early stage tumors.

\section{Methods}

\section{Patients and tissue samples}

We obtained pathologically confirmed formalin-fixed paraffin-embedded tissue specimens of 379 stages I-III CRC patients with typical adenocarcinoma histology. Of these, 228 received curative surgery in 150th Hospital of PLA (Luoyang, China) between May 2006 and October 2008 and 151 received curative surgery in Changzheng Hospital, Second Military Medical University (Shanghai, China) between July 2006 and April 2008. Distribution of the continuous variables of the two study cohorts was listed in Additional file 1. Detailed clinicopathologic features of CRC patients were listed in Table 1. The follow-up period was defined as the interval from the date of surgery to the date of death or last follow-up. The final date of follow-up was 26 September 2014 for patients from 150th Hospital of PLA (the Luoyang cohort) and 11 July 2014 for patients from Changzheng Hospital (the Shanghai cohort). Disease-specific survival (DSS) was defined as the interval from the date of surgery to the date that patient died of CRC. Patients alive at the end of follow-up were treated as censored data. Patients were excluded from the study cohorts with the following exclusion criteria: previously received any anticancer therapy; impaired heart, lung, liver, or kidney function; previous malignant disease. TNM staging was classified according to the American Joint Committee on Cancer staging manual (seventh edition).
Table 1 Clinicopathologic features of CRC patients in the training and validation cohorts

\begin{tabular}{|c|c|c|c|c|}
\hline \multirow[t]{2}{*}{ Characteristics } & \multicolumn{2}{|c|}{$\begin{array}{l}\text { Training cohort } \\
(n=228)\end{array}$} & \multicolumn{2}{|c|}{$\begin{array}{l}\text { Validation cohort } \\
(n=151)\end{array}$} \\
\hline & \multicolumn{2}{|c|}{ No. of patients (\%) } & \multicolumn{2}{|c|}{ No. of patients (\%) } \\
\hline \multicolumn{5}{|l|}{ Age (years) } \\
\hline$<60$ & 71 & $(31.1)$ & 43 & $(28.5)$ \\
\hline$\geq 60$ & 157 & $(68.9)$ & 108 & $(71.5)$ \\
\hline \multicolumn{5}{|l|}{ Sex } \\
\hline Female & 95 & $(41.7)$ & 61 & $(40.4)$ \\
\hline Male & 133 & $(58.3)$ & 90 & (59.6) \\
\hline \multicolumn{5}{|l|}{ Tumor location } \\
\hline Rectum & 169 & $(74.1)$ & 81 & (53.6) \\
\hline Colon & 59 & $(25.9)$ & 70 & $(46.4)$ \\
\hline \multicolumn{5}{|c|}{ Differentiation grade } \\
\hline Well/Moderate & 171 & $(75.0)$ & 114 & $(75.5)$ \\
\hline Poor & 57 & $(25.0)$ & 37 & $(24.5)$ \\
\hline \multicolumn{5}{|l|}{ Tumor size $(\mathrm{cm})$} \\
\hline$<5$ & 92 & $(40.4)$ & 57 & $(37.7)$ \\
\hline$\geq 5$ & 136 & $(59.6)$ & 94 & $(62.3)$ \\
\hline \multicolumn{5}{|l|}{ Local invasion } \\
\hline$T_{1}-T_{2}$ & 44 & $(19.3)$ & 14 & $(9.3)$ \\
\hline$T_{3}-T_{4}$ & 184 & $(80.7)$ & 137 & $(90.7)$ \\
\hline \multicolumn{5}{|c|}{ Lymph node metastasis } \\
\hline$N_{0}$ & 132 & $(57.9)$ & 84 & $(55.6)$ \\
\hline$N_{1}$ & 64 & $(28.1)$ & 48 & $(31.8)$ \\
\hline $\mathrm{N}_{2}$ & 32 & $(14.0)$ & 19 & $(12.6)$ \\
\hline \multicolumn{5}{|l|}{ TNM stage } \\
\hline I & 36 & $(15.8)$ & 10 & (6.6) \\
\hline$\|$ & 96 & $(42.1)$ & 74 & $(49.0)$ \\
\hline III & 96 & $(42.1)$ & 67 & $(44.4)$ \\
\hline \multicolumn{5}{|l|}{ Death } \\
\hline No & 124 & $(54.4)$ & 82 & (54.3) \\
\hline Yes & 104 & $(45.6)$ & 69 & $(45.7)$ \\
\hline
\end{tabular}

Fresh-frozen CRC samples obtained from 84 stages IIII primary CRC patients who received curative surgery in 150th Hospital of PLA from April 2013 to September 2013 were used for quantitative polymerase chain reaction (qPCR) and Western blot analysis. Written informed consent was obtained from each patient and this study was approved by the Ethical Committee of 150th Hospital of PLA and Changzheng Hospital.

\section{Real-Time qPCR analysis}

Real-Time qPCR analysis was performed as described previously [17]. Briefly, total RNAs were isolated from frozen specimens using TRIzol Reagent (Invitrogen). Reverse transcription was performed using RevertAid ${ }^{\mathrm{m}}$ 
First Strand cDNA Synthesis Kit (Thermo Scientific) according to the manufacturer's instructions. qPCR was performed on ABI Prism 7500 Sequence Detection System with SYBR Premix Ex $\mathrm{Taq}^{\mathrm{Tm}}$ II (Takara) using the $2^{-\Delta \Delta C T}$ method. Gene expression results were normalized by internal control $\beta$-actin. The primers used in this study are as follows: CA7 (NM_005182.2) forward, 5'-CTGCTTTAAGAGGCTGCTCCG-3'; reverse, 5'-CCC TGGGCAATGGGATACAG-3'; $\beta$-actin (NM_001101.3) forward, 5'-AATCGTGCGTGACATTAAGGAG-3'; reverse, 5'-ACTGTGTTGGCGT ACAGGTCTT-3'. Each sample was tested in triplicate.

\section{Western blot analysis}

Western blotting was performed as described previously [18]. Briefly, tumor specimens were prepared in lysis buffer [Tris- $\mathrm{HCl}(20 \mathrm{mM}), \mathrm{pH} 7.4, \mathrm{NaCl}(150 \mathrm{mM})$, glycerol (10\%), Nonidet P-40 (0.2\%), EDTA (1 mM), EGTA (1 mM), PMSF (1 mM), NaF (10 mM), aprotinin (5 mg/ $\mathrm{ml})$, leupeptin $(20 \mathrm{mM})$, and sodium orthovanadate $(1 \mathrm{mM})]$ and centrifuged at $12,000 \mathrm{~g}$ for $30 \mathrm{~min}$. Protein concentrations were measured using the $\mathrm{BCA}$ assay. Immunoblotting was performed using a primary antibody specific for CA7 (Abcam, ab103116) and immunocomplexes were incubated with a goat anti-rabbit fluorescein- conjugated secondary antibody, and then detected using an Odyssey fluorescence scanner (Li-Cor, Gene Company). $\beta$-actin was used as a loading control (Santa Cruz Biotechnology, sc-47778).

\section{Immunohistochemistry analysis}

Immunohistochemistry of paraffin-embedded tissue sections was performed as described previously [19]. Briefly, sections were deparaffinized and rehydrated. The endogenous peroxidase activity was blocked with $3 \% \mathrm{H}_{2} \mathrm{O}_{2}$ for 10 minutes. Antigens were retrieved with citrate buffer $\left(10 \mathrm{mM}, \mathrm{pH}\right.$ 6.0) for 15 minutes at $100^{\circ} \mathrm{C}$ in a microwave oven. After blocking, the sections were incubated with a primary anti-CA7 antibody (Abcam, ab103116) with 1:50 dilution at $4^{\circ} \mathrm{C}$ overnight in a moist chamber followed by incubated with an anti-rabbit peroxidaseconjugated secondary antibody (Santa Cruz) at room temperature for 30 minutes. Finally, the visualization signal was developed with diaminobenzidine (Dako) and the slides were counterstained with hematoxylin.

Stained sections were evaluated in a blinded manner without prior knowledge of the clinical data using the German immunoreactive score (IRS) as described previously [20]. Briefly, staining intensity was graded as "0" (negative), "1" (weak), "2" (moderate) and "3" (strong); staining extent was graded as "0" (<5\%), "1" (5-25\%), "2" (25-50\%), "3" (50-75\%) or "4" (>75\%). Values of the staining intensity and the staining extent were multiplied as a final IRS of CA7 expression. Intratumoral CA7 expression was defined as follows: low expression with the IRS $<3$ and high expression with the IRS $\geq 3$. Discrepancies in the IRS were resolved by discussing together with other pathologists to reach a consensus. Tissue samples of patients from the Luoyang cohort were used as a training set. Prognostic value of the expression of CA7 was subsequently validated in the patients from the Shanghai cohort as an external validation set.

\section{Statistical analysis}

Mann-Whitney U test was used to compare CA7 levels between groups. Pearson chi-square test or Fisher exact test was used to analyze the relationship between CA7 expression and clinical features. Kaplan-Meier analysis with log-rank test was used to compare patients' survival between subgroups. The effect of each variable on survival was determined by the Cox multivariate regression analysis. All statistical analyses were carried out using SPSS PASW Statistics 18.0 software (SPSS, Inc., Chicago, IL), and $p$ value $<0.05$ were considered to be statistically significant.

\section{Results}

\section{Downregulation of CA7 in CRC tissues}

We first examined the expression levels of CA7 mRNA in 59 paired human primary CRC tissues and adjacent normal mucosa tissues using real-time qPCR analysis. As shown in Figure 1A, CA7 mRNA expression was markedly decreased in tumor tissues compared with adjacent normal mucosa tissues $(50 / 59, \mathrm{p}<0.001)$. In addition, Western blot analysis from an independent set of 25 paired CRC and adjacent normal specimens demonstrated that protein expression of CA7 was significantly downregulated in tumor tissues compared with adjacent normal tissues $(18 / 25, \mathrm{p}<0.001$, Figure $1 B)$.

To further investigate the phenotypic expression patterns of CA7 protein in CRC tissues, IHC analysis was performed in 228 specimens of patients from the training cohort. Representative patterns of CA7 expression (negative, weak, moderate, strong) were shown in Figure $1 \mathrm{C}$. Positive staining of CA7 was mainly localized in the cytoplasm. Of note, in normal colon, the strongest immunostaining for CA7 was predominantly localized in the superficial part of the mucosa, while its expression in carcinomas was diffuse. Overall, $56.1 \%(128 / 228)$ of the adjacent normal mucosa tissues presented strong immunostaining, 35.6\% (81/228) of cases showed moderate staining, 8.3\% (19/228) showed weak staining and none showed negative staining of CA7 protein. In striking contrast, 39.9\% (91/228) of the cancerous tissues investigated showed negative immunoreactivity, $52.2 \%(119 / 228)$ of cases showed weak staining, $7.0 \%(16 / 228)$ showed moderate staining and 

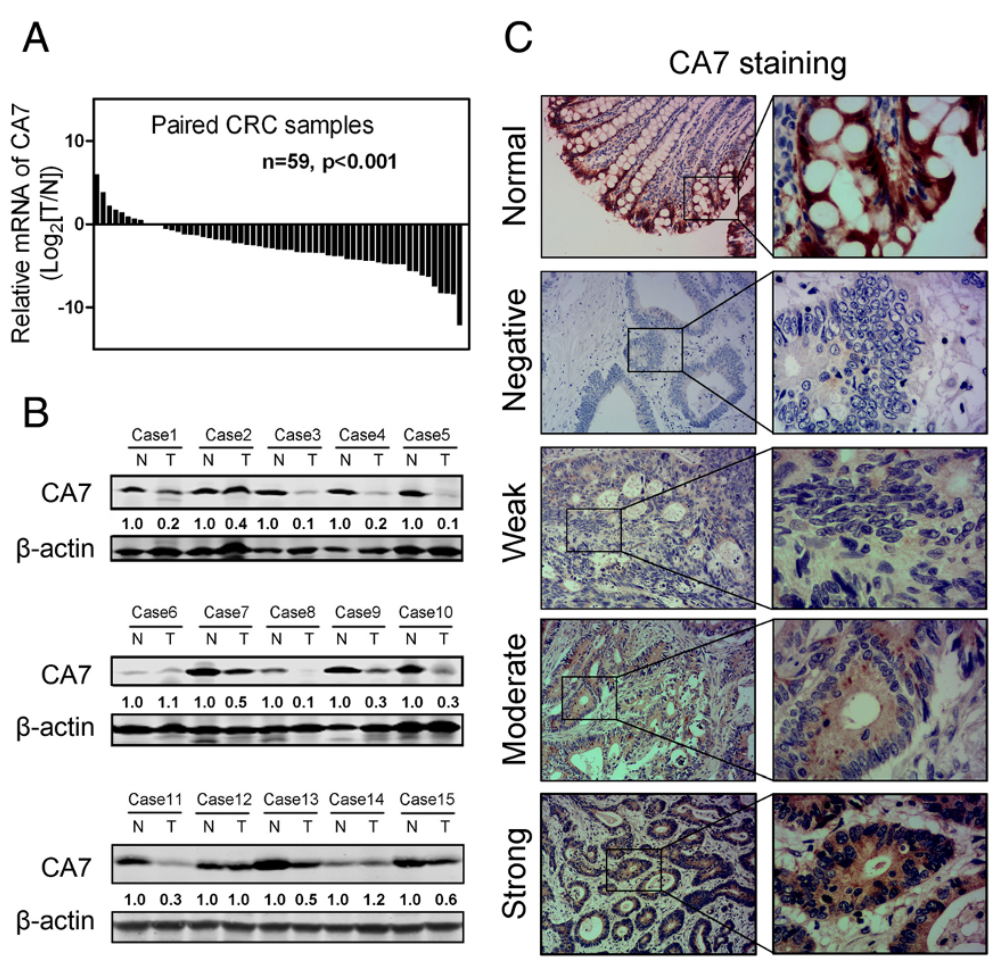

$\beta$-actin
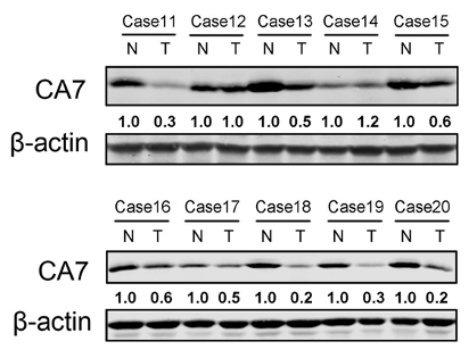

D

CA7 Staining Intensity
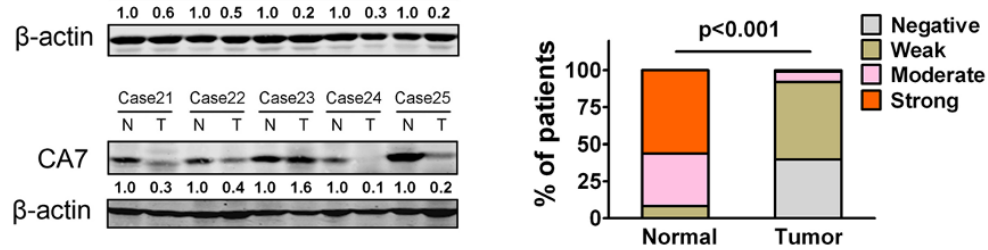

Figure 1 CA7 expression is frequently downregulated in CRC. (A) The expression levels of CA7 mRNA in 59 paired human primary CRC tissues (T) and adjacent normal tissues ( $N$ ) were evaluated by real-time qPCR methods. ( $T$ vs $N, p<0.001$ ) (B) Protein levels of CA7 in an independent set of 25 paired CRC specimens and adjacent normal tissues were determined by Western blot assay. $\beta$-actin was used as a loading control. The relative protein expression of CA7 was quantified and normalized to $\beta$-actin. Each N was arbitrarily designated 1.0. (T: Tumor; N: adjacent normal tissues, T vs $N, p<0.001)$ (C) Representative immunohistochemical expression patterns of CA7 in cancerous and adjacent normal mucosa specimens were shown. (Magnification, left panel, $\times 100$; right panel, $\times 400$ ) (D) Percentage of patients with different staining intensity of CA7 in the tumor or adjacent normal tissues in the training cohort $(p<0.001)$.

only $0.9 \%(2 / 228)$ showed relatively strong staining of CA7 ( $<<0.001$, Figure 1D). Consistently, IHC data from the validation cohort containing 151 CRC patients yielded a similar result (Additional file 2). Thus, the significant decreased staining signal for CA7 in the cancerous specimens definitely confirmed that CA7 was frequently downregulated in CRC tissues.

\section{Correlation of CA7 expression with clinicopathologic features}

Next, we evaluated the relationship between CA7 expression levels and clinicopathologic characteristics of CRC patients. Based on the immunoreactive score (IRS) of intratumoral CA7 expression, patients in the training cohort were divided into high and low CA7 expression subgroups with the median IRS value as the cut-off. As shown in Table 2, low expression of CA7 protein was significantly correlated with poor differentiation $(\mathrm{p}=0.006)$, positive lymph node metastasis $(\mathrm{p}=0.003)$, advanced TNM stage $(\mathrm{p}=0.008)$ and increased death $(\mathrm{p}<0.001)$. No significant associations were observed between CA7 expression and patient age, sex, tumor location, tumor size or local invasion.

We then applied the same cut-off to dichotomise the study patients in the validation cohort. Consistently, low levels of CA7 protein were significantly correlated with 
Table 2 Association between CA7 expression and clinicopathologic characteristics of CRC patients in the training and validation cohorts

\begin{tabular}{|c|c|c|c|c|c|c|c|c|c|c|}
\hline \multirow{4}{*}{ Characteristics } & \multicolumn{5}{|c|}{ Training cohort $(n=228)$} & \multicolumn{5}{|c|}{ Validation cohort $(n=151)$} \\
\hline & \multicolumn{4}{|c|}{ CA7 expression } & \multirow[t]{3}{*}{$\mathrm{P}_{\text {value }}{ }^{\mathrm{a}}$} & \multicolumn{4}{|c|}{ CA7 expression } & \multirow[t]{3}{*}{$P$ value } \\
\hline & \multirow{2}{*}{\multicolumn{2}{|c|}{$\begin{array}{l}\text { Low (\%) } \\
(n=116)\end{array}$}} & \multirow{2}{*}{\multicolumn{2}{|c|}{$\frac{\text { High (\%) }}{(n=112)}$}} & & \multirow{2}{*}{\multicolumn{2}{|c|}{$\begin{array}{l}\text { Low (\%) } \\
(n=89)\end{array}$}} & \multirow{2}{*}{\multicolumn{2}{|c|}{$\frac{\text { High (\%) }}{(n=62)}$}} & \\
\hline & & & & & & & & & & \\
\hline$<60$ & 40 & $(34.5)$ & 31 & $(27.7)$ & & 24 & $(27.0)$ & 19 & (30.6) & \\
\hline$\geq 60$ & 76 & $(65.5)$ & 81 & $(72.3)$ & & 65 & $(73.0)$ & 43 & $(69.4)$ & \\
\hline Sex & & & & & 0.720 & & & & & 0.490 \\
\hline Female & 47 & $(40.5)$ & 48 & $(42.9)$ & & 38 & $(42.7)$ & 23 & $(37.1)$ & \\
\hline Male & 69 & $(59.5)$ & 64 & $(57.1)$ & & 51 & $(57.3)$ & 39 & $(62.9)$ & \\
\hline Tumor location & & & & & 0.224 & & & & & 0.806 \\
\hline Rectum & 90 & $(77.6)$ & 79 & $(70.5)$ & & 47 & $(52.8)$ & 34 & $(54.8)$ & \\
\hline Colon & 26 & $(22.4)$ & 33 & $(29.5)$ & & 42 & $(47.2)$ & 28 & $(45.2)$ & \\
\hline Differentiation grade & & & & & 0.006 & & & & & 0.046 \\
\hline Well/Moderate & 78 & $(67.2)$ & 93 & $(83.0)$ & & 62 & $(69.7)$ & 52 & (83.9) & \\
\hline Poor & 38 & $(32.8)$ & 19 & $(17.0)$ & & 27 & $(30.3)$ & 10 & $(16.1)$ & \\
\hline Tumor size $(\mathrm{cm})$ & & & & & 0.448 & & & & & 0.586 \\
\hline$<5$ & 44 & $(37.9)$ & 48 & $(42.9)$ & & 32 & $(36.0)$ & 25 & $(40.3)$ & \\
\hline$\geq 5$ & 72 & $(62.1)$ & 64 & $(57.1)$ & & 57 & $(64.0)$ & 37 & $(59.7)$ & \\
\hline Local invasion & & & & & 0.141 & & & & & 0.064 \\
\hline$T_{1}-T_{2}$ & 18 & $(15.5)$ & 26 & $(23.2)$ & & 5 & (5.6) & 9 & $(14.5)$ & \\
\hline$T_{3}-T_{4}$ & 98 & $(84.5)$ & 86 & $(76.8)$ & & 84 & $(94.4)$ & 53 & $(85.5)$ & \\
\hline Lymph node metastasis & & & & & 0.003 & & & & & 0.003 \\
\hline $\mathrm{N}_{0}$ & 56 & $(48.3)$ & 76 & $(67.9)$ & & 41 & $(46.1)$ & 43 & $(69.4)$ & \\
\hline$N_{1}$ & 36 & $(31.0)$ & 28 & $(25.0)$ & & 31 & $(34.8)$ & 17 & $(27.4)$ & \\
\hline $\mathrm{N}_{2}$ & 24 & $(20.7)$ & 8 & (7.1) & & 17 & $(19.1)$ & 2 & (3.2) & \\
\hline TNM stage & & & & & 0.008 & & & & & 0.003 \\
\hline 1 & 13 & $(11.2)$ & 23 & $(20.5)$ & & 2 & $(2.2)$ & 8 & $(12.9)$ & \\
\hline$\|$ & 43 & $(37.1)$ & 53 & $(47.3)$ & & 39 & $(43.9)$ & 35 & $(56.5)$ & \\
\hline III & 60 & $(51.7)$ & 36 & $(32.2)$ & & 48 & (53.9) & 19 & (30.6) & \\
\hline Death & & & & & $<0.001$ & & & & & $<0.001$ \\
\hline No & 47 & $(40.5)$ & 77 & $(68.8)$ & & 37 & $(41.6)$ & 45 & (72.6) & \\
\hline Yes & 69 & $(59.5)$ & 35 & $(31.2)$ & & 52 & $(58.4)$ & 17 & $(27.4)$ & \\
\hline
\end{tabular}

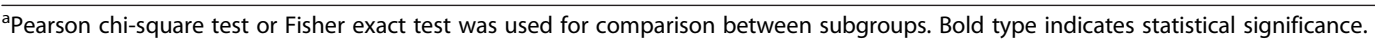

differentiation grade $(p=0.046)$, lymph node metastasis $(\mathrm{p}=0.003)$, TNM stage $(\mathrm{p}=0.003)$ and patient death $(\mathrm{p}<0.001)$. Collectively, these results indicated that intratumoral CA7 expression was negatively associated with the progression of $\mathrm{CRC}$.

\section{Prognostic values of CA7 expression in CRC patients}

Kaplan-Meier survival analyses showed that patients with low CA7 expression had significantly poorer DSS rates than those with high CA7 expression in the training cohort ( $\mathrm{p}<0.001$, Figure $2 \mathrm{~A})$. The cumulative 5 -year DSS rate was $73.2 \%$ for patients in the high-CA7-expression group, whereas it was only $44.8 \%$ for those in the lowCA7-expression group. Similarly, patients who had tumors with low CA7 expression had a significantly shorter DSS than those who had tumors with high CA7 expression in the validation cohort $(\mathrm{p}<0.001$, Figure $2 \mathrm{~B})$. In both of the two cohorts, patients who had advanced stage (stage III) tumors had a significantly worse prognosis than those who had early stage (stages I-II) 


\section{Training Cohort}

A

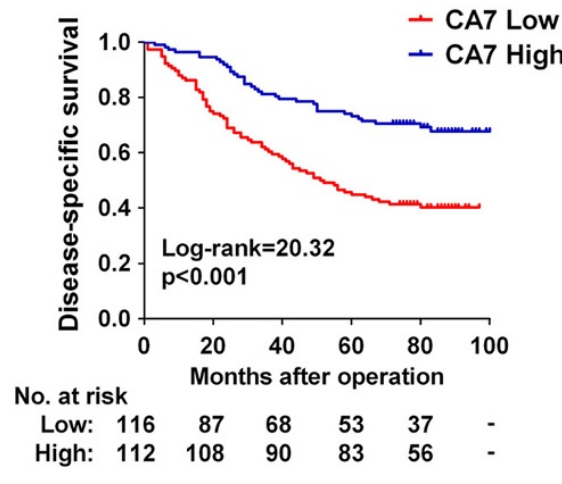

C

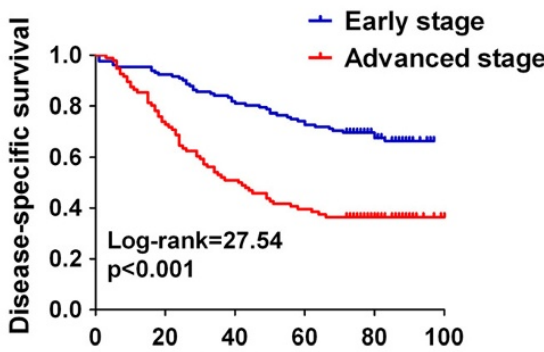

No. at risk

Months after operation

Early: $\begin{array}{lllll}132 & 123 & 108 & 98 & 67\end{array}$

Advanced: $\begin{array}{rrrrr}96 & 71 & 50 & 39 & 26\end{array}$

\section{Validation Cohort}

$\mathrm{B}$

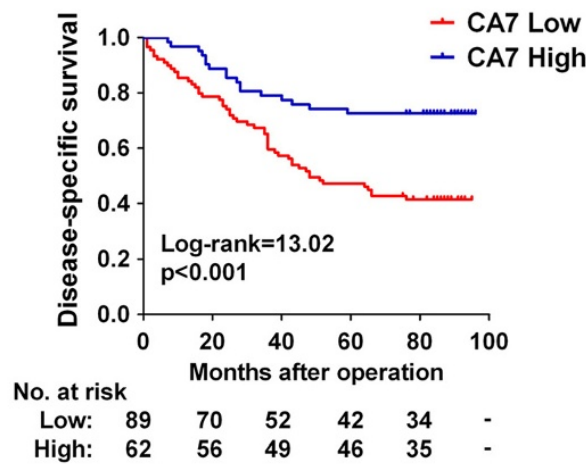

D

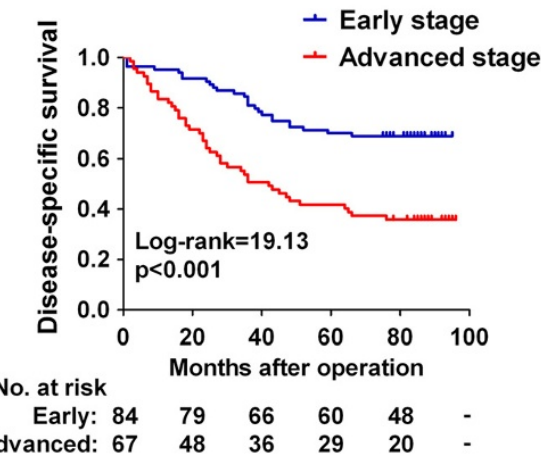

Figure 2 Kaplan-Meier survival analysis for CRC patients in the training and validation cohorts. (A-B) Kaplan-Meier curves for diseasespecific survival of CRC patients in the training (A) and validation (B) cohorts according to CA7 expression status. Patients were divided into high and low CA7 expression subgroups with the median IRS value as the cut-off. (C-D) Kaplan-Meier curves for disease-specific survival of CRC patients in the training $(\mathbf{C})$ and validation (D) cohort according to TNM stage of the disease. The p-value was determined using the log-rank test.

tumors (all $\mathrm{p}<0.001$, Figure $2 \mathrm{C}$ and $\mathrm{D}$ ). These data were consistent with the well established adverse prognostic effect of tumor stage [21] and confirmed that our cohorts were representative and that the survival analyses were valid. Importantly, reduced expression of CA7 significantly predicted poor DSS for patients with early stage tumors both in the training $(\mathrm{p}=$ 0.012 , Figure $3 \mathrm{~A})$ and validation cohorts $(\mathrm{p}=0.002$, Figure $3 \mathrm{~B}$ ). In addition, low levels of CA7 protein also predicted unfavorable DSS for patients with advanced stage tumors in the training cohort $(p=0.004$, Figure 3C). While, the survival difference was not statistically significant for patients with advanced stage tumors in the validation cohort $(p=0.289$, Figure 3D).

The independent prognostic significance of CA7 expression on CRC-specific survival was assessed with a Cox regression model. The clinicopathologic variables considered to be potential predictors of survival were shown in Table 3. Univariate analyses indicated that factors including patient age, tumor differentiation grade, TNM stage and CA7 expression were predictors of DSS both in the training and validation cohort. The factors that significantly correlated with survival in the univariate analysis were further assessed by multivariate analysis. The results revealed that, besides the patient age and TNM stage of the disease, decreased CA7 expression was an independent adverse prognostic factor not only in the training cohort (HR, 2.247; 95\% CI, 1.481-3.401, $\mathrm{p}<0.001)$ but also in the validation cohort (HR, 2.058; 95\% CI, 1.174-3.610, $\mathrm{p}=0.012$ ).

More importantly, stage-based survival analyses revealed that decreased expression of CA7 also was an independent predictor of poor prognosis for patients with early stage tumors in the training cohort (HR, 2.232; 95\% CI, 1.220-4.082, $\mathrm{p}=0.009$ ) as well as in the validation cohort $(\mathrm{HR}, 3.165 ; 95 \% \mathrm{CI}, 1.326-7.519$, 


\section{Training Cohort}

A

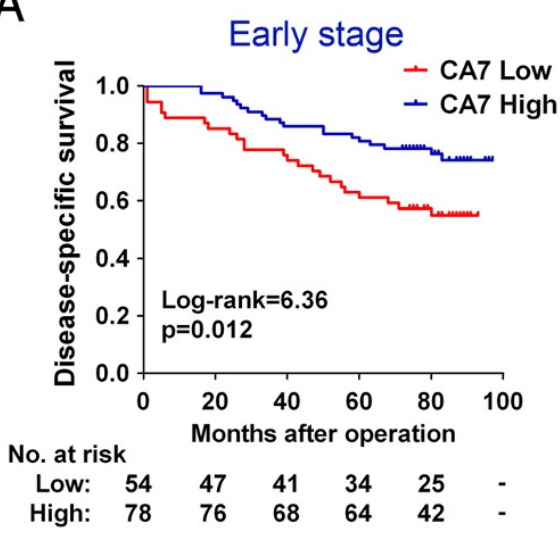

C

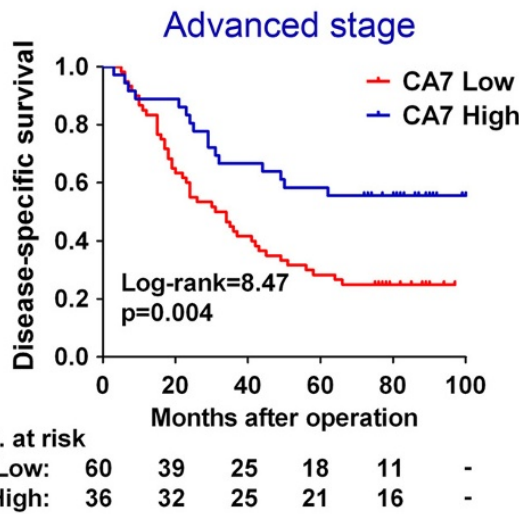

Validation Cohort
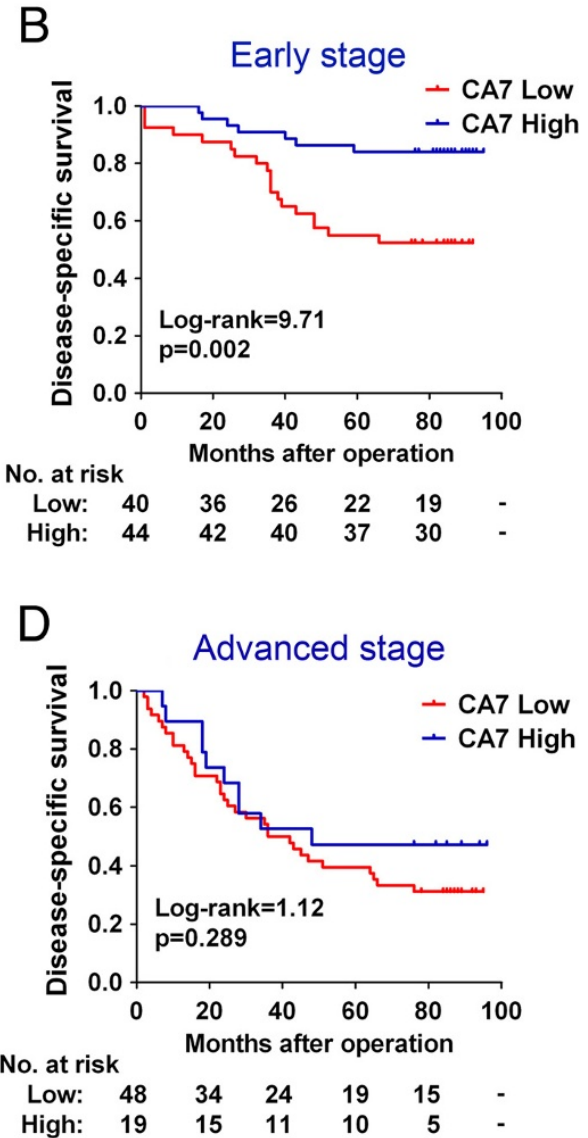

Figure 3 Kaplan-Meier survival analysis for CRC patients with early or advanced stage tumors in the training and validation cohorts. (A-B) Kaplan-Meier curves for disease-specific survival of CRC patients with early stage tumors in the training (A) and validation (B) cohorts according to CA7 expression status. (C-D) Kaplan-Meier curves for disease-specific survival of CRC patients with advanced stage tumors in the training $(\mathbf{C})$ and validation (D) cohorts according to CA7 expression status. The p-value was determined using the log-rank test.

$\mathrm{p}=0.009$ ) (Table 4). In addition, low CA7 expression was also independently associated with poor DSS for patients with advanced stage tumors in the training cohort (HR, 2.809; 95\% CI, 1.524-5.181, $\mathrm{p}=0.001$ ). However, the prognostic significance of CA7 was not statistically significant for patients with advanced stage tumors in the validation cohort. Taken together, these data demonstrated that decreased CA7 expression was closely related to poor patient survival, especially for those with early stage tumors.

\section{Discussion}

To date, CAs have been implicated in the tumorigenesis of several human malignancies including CRC during the past decades. It has been shown that CA I, II and XIII are downregulated while CA IX and XII are upregulated in the cancerous tissue compared with the normal colorectal epithelium [7-12]. In addition, prognostic implications of individual CA isozymes in CRC have also been demonstrated. For instance, CA I positive immunostaining has been associated with well or moderate differentiation, lack vascular invasion and favorable clinical outcome of CRC [7,22], whereas, high expression of CA IX has been linked with worse prognosis of CRC patients [23-25]. Thus, distinct tumor-associated CAs apparently have different expression patterns and prognostic significances in CRC.

Recently, attention has been focus on the CA7 because of its high catalytic activity and relatively limited tissue distribution $[13,26]$. It has been shown that CA7 is expressed in several organs including brain, stomach, duodenum, colon, liver, and skeletal muscle. In addition, the expression of CA7 and its prognostic significance has been demonstrated in human diffuse astrocytomas [16]. However, its clinical relevance has not been assessed in CRC. Although a previous gene 
Table 3 Univariate and multivariate analyses of CA7 expression and disease-specific survival of patients in the training and validation cohorts

\begin{tabular}{|c|c|c|c|c|c|c|c|}
\hline \multirow[t]{2}{*}{ Variables } & \multirow[t]{2}{*}{ Categories } & \multicolumn{3}{|c|}{ Univariate analysis } & \multicolumn{3}{|c|}{ Multivariate analysis $^{\mathbf{b}}$} \\
\hline & & HR & $95 \% \mathrm{Cl}$ & P value ${ }^{c}$ & HR & $95 \% \mathrm{Cl}$ & $P$ value \\
\hline \multicolumn{8}{|l|}{ Training Cohort } \\
\hline Age (years) & $\geq 60 /<60$ & 2.263 & $1.389-3.687$ & 0.001 & 2.770 & $1.691-4.537$ & $<0.001$ \\
\hline Sex & Male / female & 0.878 & $0.596-1.293$ & 0.510 & & & \\
\hline Tumor location & Colon / rectum & 1.401 & $0.923-2.125$ & 0.113 & & & \\
\hline Tumor size $(\mathrm{cm})$ & $\geq 5 /<5$ & 1.114 & $0.753-1.648$ & 0.590 & & & \\
\hline Differentiation grade & Poor / well + moderate & 2.626 & $1.761-3.916$ & $<0.001$ & 2.010 & $1.337-3.023$ & 0.001 \\
\hline TNM stage & $\||| / \mid+\|$ & 2.734 & $1.846-4.048$ & $<0.001$ & 2.402 & $1.603-3.598$ & $<0.001$ \\
\hline CA7 expression ${ }^{a}$ & Low / high & 2.469 & $1.642-3.717$ & $<0.001$ & 2.247 & $1.481-3.401$ & $<0.001$ \\
\hline \multicolumn{8}{|l|}{ Validation Cohort } \\
\hline Age (years) & $\geq 60 /<60$ & 1.976 & $1.080-3.614$ & 0.027 & 2.293 & $1.247-4.217$ & 0.008 \\
\hline Sex & Male / female & 0.737 & $0.459-1.183$ & 0.207 & & & \\
\hline Tumor location & Colon / rectum & 0.961 & $0.599-1.542$ & 0.868 & & & \\
\hline Tumor size $(\mathrm{cm})$ & $\geq 5 /<5$ & 0.814 & $0.503-1.317$ & 0.403 & & & \\
\hline Differentiation grade & Poor / well + moderate & 1.688 & $1.017-2.803$ & 0.043 & & & \\
\hline TNM stage & ||$|/|+\|$ & 2.828 & $1.734-4.611$ & $<0.001$ & 2.674 & $1.615-4.428$ & $<0.001$ \\
\hline CA7 expression ${ }^{a}$ & Low / high & 2.632 & $1.520-4.545$ & 0.001 & 2.058 & $1.174-3.610$ & 0.012 \\
\hline
\end{tabular}

Abbreviations: HR hazard ratio, 95\% Cl 95\% confidence interval.

${ }^{a}$ For CA7, median values were used as the cut-off point for definition of subgroups (low expression and high expression groups).

${ }^{\mathrm{b}}$ Multivariate models were adjusted for age, sex, tumor location, tumor size, differentiation grade, and TNM stage.

'Bold type indicates statistical significance.

Table 4 Multivariate analyses of CA7 expression and disease-specific survival for patients with early or advanced stage tumors in the training and validation cohorts

\begin{tabular}{|c|c|c|c|c|c|c|c|}
\hline \multirow[t]{2}{*}{ Variables } & \multirow[t]{2}{*}{ Categories } & \multicolumn{3}{|c|}{ Early stage } & \multicolumn{3}{|c|}{ Advanced stage } \\
\hline & & $\overline{\mathrm{HR}}$ & $95 \% \mathrm{Cl}$ & $\mathrm{P}$ value ${ }^{\mathrm{b}}$ & $\overline{\mathrm{HR}}$ & $95 \% \mathrm{Cl}$ & $\mathrm{P}$ value \\
\hline \multicolumn{8}{|l|}{ Training Cohort } \\
\hline Age (years) & $\geq 60 /<60$ & 4.062 & $1.592-10.365$ & 0.003 & 2.353 & $1.282-4.319$ & 0.006 \\
\hline Sex & Male / female & & & & & & \\
\hline Tumor location & Colon / rectum & & & & & & \\
\hline Tumor size (cm) & $\geq 5 /<5$ & & & & & & \\
\hline Differentiation grade & Poor / well + moderate & & & & 2.389 & $1.419-4.023$ & 0.001 \\
\hline CA7 expression ${ }^{a}$ & Low / high & 2.232 & $1.220-4.082$ & 0.009 & 2.809 & $1.524-5.181$ & 0.001 \\
\hline \multicolumn{8}{|l|}{ Validation Cohort } \\
\hline Age (years) & $\geq 60 /<60$ & & & & 2.374 & $1.176-4.794$ & 0.016 \\
\hline Sex & Male / female & & & & & & \\
\hline Tumor location & Colon / rectum & & & & & & \\
\hline Tumor size $(\mathrm{cm})$ & $\geq 5 /<5$ & & & & & & \\
\hline Differentiation grade & Poor / well + moderate & & & & 2.637 & $1.414-4.920$ & 0.002 \\
\hline CA7 expression ${ }^{a}$ & Low / high & 3.165 & $1.326-7.519$ & 0.009 & & & \\
\hline
\end{tabular}

Abbreviations: HR hazard ratio, 95\% Cl 95\% confidence interval.

${ }^{a}$ For CA7, median values were used as the cut-off point for definition of subgroups (low expression and high expression groups).

${ }^{b}$ Bold type indicates statistical significance.

Multivariate models were adjusted for age, sex, tumor location, tumor size and differentiation grade. 
expression profiling study and a recent bioinformaticsbased analysis revealed that CA7 mRNA was downregulated in CRC clinical specimens $[14,15]$, neither of the two studies conducted validation experiments. To get a better insight into the phenotypic expression and prognostic significance of CA7 in CRC, we comprehensively analyzed both colorectal neoplasias and matched adjacent normal mucosa specimens from the same patients in two independent study cohorts.

Using qPCR and Western blot analysis, we demonstrated that CA7 was significantly downregulated in primary CRC samples at both the mRNA and protein levels. Strikingly, subsequent immunohistochemical analysis of CRC specimens from the training and validation cohorts showed that $56.1 \%$ and $66.9 \%$ of the adjacent normal mucosa sections presented strong CA7 immunoreactivity, whereas only $0.9 \%$ and $1.9 \%$ of the CRC sections showed relatively strong staining, respectively. These results definitely confirmed the significant downregulation of CA7 in CRC. Thus, the expression pattern of CA7 was generally similar with that of CA I, II and XIII in CRC $[8,12]$. While, the molecular basis for CA7 downregulation in $\mathrm{CRC}$ remains unclear and requires further investigation.

In the present study, we observed that the strongest CA7 immunoreaction was localized primarily in the mature superficial enterocytes but the signals reduced significantly along with increasing malignancy grades, indicating an association between CA7 expression and the differentiation of colorectal epithelium. In fact, several CA isozymes have been proposed to relate to differentiation. Bekku S et al. showed that CA I and II could be a differentiation marker of human and rat colonic enterocytes [27]. In addition, Leppilampi $\mathrm{M}$ et al. revealed that high expression of CA IX was associated with a differentiated phenotype of gastric epithelial cells [28]. Although our data demonstrated a correlation between CA7 expression and cell differentiation, its potential involvement in differentiation needs to be carefully determined, for the decrease in CA7 expression in less differentiated tissues could also be the result of other factors that lead to dedifferentiation, rather than downregulation of CA7 being a contributing factor in dedifferentiation. Nevertheless, regardless of the mechanism, our data indicates that CA7 might be useful in the histopathological grading of CRC.

Intriguingly, correlation analyses with clinicopathologic features from the two independent cohorts unanimously revealed a significant association between decreased CA7 expression and increased lymph node metastasis, advanced TNM stage and increased patient death, indicating that CA7 might be negatively involved in CRC progression. Recently, Monti SM and his groups have demonstrated that, apart from its canonical catalytic action of carbon dioxide hydration, CA7 has the ability to protect cells against oxidative damage [29,30]. Given that oxidative stress has been implicated in the pathogenesis of a wide spectrum of human cancers including CRC [31-34], the reported protective role of CA7 against oxidative stress might support a tumor-suppressing function for this enzyme. However, whether or not CA7 plays a functional role in the tumorigenesis and progression of CRC remains to be determined. Further studies using gain-of-function and loss-of-function strategies are warranted to address this issue.

The most interesting findings of this study are from the survival analysis results. Reduced expression of CA7 was associated with shortened survival for CRC patients not only in the training cohort but also in the external validation cohort. In univariate analysis, CA7 protein emerged as a significant prognostic factor of clinical outcome. Moreover, in multivariate analysis, it emerged as a significant independent predictor of survival in addition to tumor stage and patient age. The present study indicated that TNM stage also is an important prognostic factor in CRC, which is in agreement with its well established adverse prognostic effect [21]. Generally speaking, CRC patients who had early stage tumors (stages I-II) have a relatively favorable prognosis than those who had advanced stage tumors (stages III-IV). However, a subgroup of patients with early stage tumors have an increased risk of early recurrence and death. The potential mechanisms for these aggressive forms of early stage tumors are complicated; nevertheless, identifying this high-risk subgroup of patients would be of particular importance in the selection of patients for appropriate treatment. To further determine the prognostic value of CA7 in the therapeutic decision-making, we performed survival analyzes stage by stage. Importantly, reduced CA7 expression significantly predicted poor postoperative prognosis of patients with early stage tumors in both cohorts. More importantly, stage-based multivariate analyses from the two cohorts unanimously confirmed that decreased expression of CA7 also was an independent unfavorable prognostic indicator for patients with early stage tumors. Although we also observed a significant association between decreased CA7 expression and poor DSS in the advanced-stage patient group from the training cohort, its prognostic performance did not persist in the patients of the same stage category from the validation cohort (Figure 3D and Table 4). However, additional larger validations will be needed to further assess the prognostic significance of CA7 expression in patients with advance stage tumors. Of note, in contrast to our results, Bootorabi $\mathrm{F}$ et al. reported that high CA7 expression correlates with poor prognosis of patients with astrocytomas [16]. The discrepant results on the prognostic value of CA7 in different malignances 
indicate that its prognostic implication may be tissuedependent and varies with the type of malignancy. The underlying mechanism for the prognostic importance of CA7 in CRC is currently unknown and needs to be further investigated. Nevertheless, our results suggest that determination of the intratumoral CA7 expression status may help to identify patients with aggressive forms of $\mathrm{CRCs}$ and further guide individualized therapy choices.

The present study remains to be improved on several aspects. Although we enrolled consecutive patients in the training cohort, some of them were lost to followup, which may introduce a bias. In addition, disease recurrence monitoring was incomplete in the two patient cohorts, resulting in the loss of disease-free survival data, which is also very important for a biomarker validation. Further prospective studies using large cohorts are necessary to validate the robustness of our findings before clinical translation.

\section{Conclusions}

In summary, this study is the first to demonstrate that CA7 is frequently downregulated in CRC and that decreased expression of CA7 is closely related to aggressive clinical features and poor postoperative prognosis of CRC patients. Our findings from two independent cohorts provide evidence for the potential utility of CA7 as a prognostic marker for patients with CRC, especially for those with early stage tumors. In addition, results from the present work encourage further investigation of its potential role in CRC pathobiology.

\section{Additional files}

Additional file 1: Distribution of the continuous variables of the two study cohorts.

Additional file 2: Percentage of patients with different staining intensity of CA7 in the tumor or adjacent normal tissues in the validation cohort.

\section{Abbreviations}

CRC: Colorectal carcinoma; CA7: Carbonic anhydrase VII; DSS: Diseasespecific survival; QPCR: Quantitative polymerase chain reaction; IHC: Immunohistochemistry; IRS: Immunoreactive score.

\section{Competing interests}

The authors declare that they have no competing interests.

\section{Authors' contributions}

GZY and LH performed the immunohistochemistry and interpreted the IHC results, GZY, LH and JC performed the statistical analysis, JC, HYC, YZ, DF and YXZ obtained the clinical parameters and survival information, GZY, LH and HYC performed the Western blot analysis, GZY, CYQ, HG and HF performed the APCR analysis, GZY, LH, QPC and CFG conceived of the study, and participated in its design and coordination. GZY and LH drafted the manuscript. All authors read and approved the final manuscript.

\section{Acknowledgements}

This work was supported by National Natural Science Foundation of China (81301811).

\section{Author details}

'Anal-Colorectal Surgery Institute, 150th Hospital of PLA, Luoyang, China. 2Department of Clinical Laboratory, 150th Hospital of PLA, Luoyang, China ${ }^{3}$ Department of Colorectal Surgery, 150th Hospital of PLA, Luoyang, China. ${ }^{4}$ Department of Oncology, 150th Hospital of PLA, Luoyang, China. ${ }^{5}$ Department of Oncology, Changhai Hospital, Second Military Medical University, Shanghai, China. ${ }^{6}$ Department of Gastrointestine Surgery, Changzheng Hospital, Second Military Medical University, Shanghai, China.

Received: 11 November 2014 Accepted: 18 March 2015

Published online: 01 April 2015

\section{References}

1. Jemal A, Bray F, Center MM, Ferlay J, Ward E, Forman D. Global cancer statistics. CA Cancer J Clin. 2011;61:69-90.

2. Ferlay J, Shin HR, Bray F, Forman D, Mathers C, Parkin DM. Estimates of worldwide burden of cancer in 2008: GLOBOCAN 2008. Int J Cancer. 2010;127:2893-917.

3. Brenner H, Kloor M, Pox CP. Colorectal cancer. Lancet. 2014;383:1490-502.

4. Guo P, Huang ZL, Yu P, Li K. Trends in cancer mortality in China: an update. Ann Oncol. 2012;23:2755-62.

5. Alterio V, Di Fiore A, D'Ambrosio K, Supuran CT, De Simone G. Multiple binding modes of inhibitors to carbonic anhydrases: how to design specific drugs targeting 15 different isoforms? Chem Rev. 2012;112:4421-68.

6. Supuran CT. Carbonic anhydrases: novel therapeutic applications for inhibitors and activators. Nat Rev Drug Discov. 2008;7:168-81.

7. Mori M, Staniunas RJ, Barnard GF, Jessup JM, Steele Jr GD, Chen LB. The significance of carbonic anhydrase expression in human colorectal cancer. Gastroenterology. 1993;105:820-6.

8. Kivela AJ, Saarnio J, Karttunen TJ, Kivelä J, Parkkila AK, Pastorekova S, et al. Differential expression of cytoplasmic carbonic anhydrases, CA I and II, and membrane-associated isozymes, CA IX and XII, in normal mucosa of large intestine and in colorectal tumors. Dig Dis Sci. 2001;46:2179-86.

9. Saarnio J, Parkkila S, Parkkila AK, Haukipuro K, Pastoreková S, Pastorek J, et al. Immunohistochemical study of colorectal tumors for expression of a novel transmembrane carbonic anhydrase, MN/CA IX, with potential value as a marker of cell proliferation. Am J Pathol. 1998;153:279-85.

10. Niemelä AM, Hynninen P, Mecklin JP, Kuopio T, Kokko A, Aaltonen L, et al. Carbonic anhydrase IX is highly expressed in hereditary nonpolyposis colorectal cancer. Cancer Epidemiol Biomarkers Prev. 2007;16:1760-6.

11. Kivelä A, Parkkila S, Saarnio J, Karttunen TJ, Kivelä J, Parkkila AK, et al. Expression of a novel transmembrane carbonic anhydrase isozyme XII in normal human gut and colorectal tumors. Am J Pathol. 2000;156:577-84.

12. Kummola L, Hämäläinen JM, Kivelä J, Kivelä AJ, Saarnio J, Karttunen T, et al. Expression of a novel carbonic anhydrase, CA XIII, in normal and neoplastic colorectal mucosa. BMC Cancer. 2005;5:41.

13. Bootorabi F, Jänis J, Smith E, Waheed A, Kukkurainen S, Hytönen V, et al. Analysis of a shortened form of human carbonic anhydrase VII expressed in vitro compared to the full-length enzyme. Biochimie. 2010;92:1072-80.

14. Birkenkamp-Demtroder K, Olesen SH, Sørensen FB, Laurberg S, Laiho P, Aaltonen $L A$, et al. Differential gene expression in colon cancer of the caecum versus the sigmoid and rectosigmoid. Gut. 2005;54:374-84.

15. Chu CM, Yao CT, Chang YT, Chou HL, Chou YC, Chen KH, et al. Gene expression profiling of colorectal tumors and normal mucosa by microarrays meta-analysis using prediction analysis of microarray, artificial neural network, classification, and regression trees. Dis Markers. 2014;2014:634123.

16. Bootorabi F, Haapasalo J, Smith E, Haapasalo H, Parkkila S. Carbonic anhydrase VII-a potential prognostic marker in gliomas. Health. 2011;3:6-12.

17. Hu L, Chen L, Yang G, Li L, Sun H, Chang Y, et al. HBx sensitizes cells to oxidative stress-induced apoptosis by accelerating the loss of MCl-1 protein via caspase-3 cascade. Mol Cancer. 2011;10:43.

18. Dong LW, Yang GZ, Pan YF, Chen Y, Tan YX, Dai RY, et al. The oncoprotein p28GANK establishes a positive feedback loop in $\beta$-catenin signaling. Cell Res. 2011;21:1248-61.

19. Li L, Chen L, Hu L, Liu Y, Sun HY, Tang J, et al. Nuclear factor high-mobility group box 1 mediating the activation of Toll-like receptor 4 signaling in hepatocytes in the early stage of nonalcoholic fatty liver disease in mice. Hepatology. 2011;54:1620-30. 
20. Tang L, Tan YX, Jiang BG, Pan YF, Li SX, Yang GZ, et al. The prognostic significance and therapeutic potential of hedgehog signaling in intrahepatic cholangiocellular carcinoma. Clin Cancer Res. 2013;19:2014-24.

21. Compton CC. Optimal pathologic staging: defining stage II disease. Clin Cancer Res. 2007;13:6862s-70.

22. Peng $Y$, Li X, Wu M, Yang J, Liu M, Zhang W, et al. New prognosis biomarkers identified by dynamic proteomic analysis of colorectal cancer. Mol Biosyst. 2012;8:3077-88.

23. Korkeila E, Talvinen K, Jaakkola PM, Minn H, Syrjänen K, Sundström J, et al. Expression of carbonic anhydrase IX suggests poor outcome in rectal cancer. Br J Cancer. 2009;24:874-80.

24. Cleven AH, van Engeland M, Wouters BG, de Bruïne AP. Stromal expression of hypoxia regulated proteins is an adverse prognostic factor in colorectal carcinomas. Cell Oncol. 2007;29:229-40.

25. Pastorekova S, Parkkila S, Zavada J. Tumor-associated carbonic anhydrases and their clinical significance. Adv Clin Chem. 2006;42:167-216.

26. Earnhardt JN, Qian M, Tu C, Lakkis MM, Bergenhem NC, Laipis PJ, et al. The catalytic properties of murine carbonic anhydrase VII. Biochemistry. 1998;37:10837-45

27. Bekku S, Mochizuki H, Takayama E, Shinomiya N, Fukamachi H, Ichinose M, et al. Carbonic anhydrase I and II as a differentiation marker of human and rat colonic enterocytes. Res Exp Med (Berl). 1998;198:175-85.

28. Leppilampi M, Saarnio J, Karttunen TJ, Kivelä J, Pastoreková S, Pastorek J, et al. Carbonic anhydrase isozymes IX and XII in gastric tumors. World J Gastroenterol. 2003;9:1398-403.

29. Del Giudice R, Monti DM, Truppo E, Arciello A, Supuran CT, De Simone G, et al. Human carbonic anhydrase VII protects cells from oxidative damage. Biol Chem. 2013:394:1343-8.

30. Truppo E, Supuran CT, Sandomenico A, Vullo D, Innocenti A, Di Fiore A, et al. Carbonic anhydrase VII is S-glutathionylated without loss of catalytic activity and affinity for sulfonamide inhibitors. Bioorg Med Chem Lett. 2012:22:1560-4.

31. Sosa $V$, Moliné $T$, Somoza $R$, Paciucci $R$, Kondoh $H$, LLeonart ME. Oxidative stress and cancer: an overview. Ageing Res Rev. 2013;12:376-90.

32. Fang J, Seki T, Maeda $H$. Therapeutic strategies by modulating oxygen stress in cancer and inflammation. Adv Drug Deliv Rev. 2009;61:290-302.

33. Perše M. Oxidative stress in the pathogenesis of colorectal cancer: cause or consequence? Biomed Res Int. 2013;2013:725710.

34. Stone WL, Krishnan K, Campbell SE, Palau VE. The role of antioxidants and pro-oxidants in colon cancer. World J Gastrointest Oncol. 2014;6:55-66.

\section{Submit your next manuscript to BioMed Central and take full advantage of:}

- Convenient online submission

- Thorough peer review

- No space constraints or color figure charges

- Immediate publication on acceptance

- Inclusion in PubMed, CAS, Scopus and Google Scholar

- Research which is freely available for redistribution 